\title{
Corrección del pene curvo en cirugía mayor ambulatoria
}

\author{
P. Navalón Verdejo, C. Zaragozá Fernández*, F. Sánchez Ballester, L. de la Torre Abril, \\ F. Ordoño Domínguez, J.J. Escudero, F. Ramada Benlloch
}

Servicio de Urología. *Unidad de CMA. Consorcio Hospital General Universitario. Valencia.

Actas Urol Esp 2005; 29 (2): 217-222

\section{RESUMEN}

CORRECCION DEL PENE CURVO EN CIRUGIA MAYOR AMBULATORIA

Objetivo: Evaluar los resultados obtenidos en la corrección del pene curvo mediante la técnica de Nesbit durante los cuatro primeros años de integración de nuestro Servicio en la Unidad de Cirugía Mayor Ambulatoria (CMA) de nuestro Hospital.

Pacientes y método: Desde enero de 2000 hasta abril de 2004 hemos intervenido en régimen ambulatorio a 21 pacientes afectos de pene curvo (12 congénito y 9 con enfermedad de Peyronie) mediante la técnica de Nesbit. Se describe el procedimiento quirúrgico-anestésico realizado y también se evalúan los criterios de inclusión y alta, así como los resultados obtenidos y el grado de satisfacción mediante la elaboración de un cuestionario.

Resultados: Ninguno de los pacientes precisó ingreso por complicación intra ni postoperatoria. Así, hemos tenido una nula incidencia de complicaciones a excepción del inevitable acortamiento del pene, claramente independiente del régimen de ambulatorización del proceso. El grado de satisfacción con el tratamiento recibido ha sido superior al 95\%.

Conclusiones: La práctica totalidad de los pacientes susceptibles de corrección quirúrgica de incurvación peneana son candidatos a ser incluidos en un programa de CMA, mejorando ostensiblemente la relación costo-eficacia, no disminuyendo por ello la calidad asistencial ni el grado de satisfacción de los pacientes.

Palabras Clave: Cirugía Mayor Ambulatoria. Pene curvo. Técnica de Nesbit.

\section{ABSTRACT}

\section{CORRECTION OF THE PENILE CURVATURE IN AMBULATORY SURGERY}

Objective: To evaluate the results obtained in the correction of the curved penis by means of Nesbit's technique during the four first years of integration of our Service in the Major Ambulatory Surgery Unit (CMA) of our Hospital.

Patients and method: From January of 2000 to April of 2004 we intervened in ambulatory regime 21 patient suffering from curved penis (12 congenital and 9 with Peyronie's disease) by means of Nesbit's technique. The surgical-anesthetic performed procedure is described and also the criteria of inclusion and discharge are evaluated, as well as the results obtained and the degree of satisfaction by means of the elaboration of a questionnaire.

Results: None of the patients needed entrance for intrasurgery nor postsurgery complications. Thus, we obtained a null incidence of complications with the exception of the inevitable shortening of the penis, clearly independent from the regime of out-patient's process. The degree of satisfaction with the received treatment has been superior to $95 \%$.

Conclusions: The practical totality of the susceptible patients for surgical correction of penile curvature are candidates to be included in a CMA program, improving obviously the relation cost-efficacy, not diminishing for that reason the welfare quality nor the degree of patients's satisfaction. 
$\mathrm{L}^{2}$ a Cirugía Mayor Ambulatoria (CMA) se define como la atención a procesos susceptibles de cirugía realizada con anestesia general, local, regional o sedación que requieren cuidados postoperatorios poco intensivos y de corta duración, por lo que el paciente no necesita ingreso hospitalario y puede ser dado de alta pocas horas después del procedimiento ${ }^{1}$, lo que implica, por lo tanto, que el paciente pasa las noches anterior y posterior a la intervención quirúrgica en su domicilio.

El incremento del nivel cultural, del denominado "estado de bienestar", y de la longevidad de la población, junto a una mayor oferta asistencial, comportan un progresivo aumento de la demanda quirúrgica, que ha puesto en evidencia, entre otras, la limitación de los recursos sanitarios. La CMA surge pues con la finalidad de optimizar estos recursos, no solo agilizando los actos quirúrgicos y racionalizando los crecientes gastos sanitarios, sino también mejorando la calidad asistencial $^{2,3}$.

La urología es una especialidad con una gran proyección en el campo de la CMA, ya que por una parte cuenta con una gran cantidad de patología de mediana complejidad susceptible de ser intervenida sin ingreso, y por otra, se trata de una especialidad que en los últimos años ha presenciado una progresiva y espectacular mejoría de su arsenal tecnológico (endoscopia, ondas de choque, hipertermia y termoterapia, láser, etc.), lo que ha propiciado una evolución hacia procedimientos quirúrgicos cada vez menos invasivos, más simples y seguros, y por lo tanto con menos necesidad de hospitalización ${ }^{4}$.

Aunque la cifra de tratamientos urológicos incluidos en la Guía de Organización y Funcionamiento para la CMA editada por el Ministerio de Sanidad y Consumo ${ }^{1}$ o por el Royal College of Surgeons se limitan a la vasectomía, cistoscopia, dilataciones uretrales, litotricia extracorpórea, circuncisión, tratamiento de lesiones escrotales, orquidectomía, cirugía del hidrocele, orquidopexia y meatoplastia, esta lista parece escasa comparándola con la diversidad descrita en la literatura y en nuestra propia experiencia ${ }^{5}$.

Siguiendo con estas directrices, y en un intento de unificar criterios, la "Conselleria de Sanitat" de la "Generalitat Valenciana" ${ }^{6}$ publicó reciente- mente una relación de procesos quirúrgicos urológicos susceptibles de ser intervenidos mediante CMA, que incluye mas de 30 tipos distintos de intervenciones, tanto de patología inguinoescrotal, peneana, uroginecológica, como de procedimientos endoscópicos y percutáneos ${ }^{5}$.

La corporoplastia de Nesbit ha demostrado ser una intervención quirúrgica sencilla y efectiva para la corrección de la incurvación peneana, tanto congénita como adquirida, siendo sin duda esta intervención ${ }^{7}$ y sus múltiples variantes descritas posteriormente $\mathrm{e}^{8,9}$ la mas ampliamente difundida para el tratamiento de esta entidad.

El procedimiento de Nesbit consiste básicamente en la exéresis de uno o varios fragmentos elipsoidales de túnica albugínea en el punto contralateral a la incurvación y su posterior sutura. Esta técnica ha sido ampliamente utilizada por numerosos autores con excelentes resultados en las incurvaciones peneanas congénitas, siendo los resultados algo peores, por lo general, en los pacientes afectos de enfermedad de Peyronie, fundamentalmente por el leve acortamiento que supone esta técnica en un paciente en el que el pene ya se encuentra acortado por la propia enfermedad $^{10}$.

Creemos que ésta técnica cumple todos los criterios necesarios para ser considerada como un procedimiento quirúrgico susceptible de ser incluido en un programa de CMA. Por lo general, se trata de pacientes jóvenes, sexualmente activos, en los que el riesgo quirúrgico-anestésico suele ser bajo o nulo. Asimismo, la técnica cumple todos los criterios de inclusión recomendados en la Guía de Organización y Funcionamiento para CMA del Ministerio de Sanidad y Consumo ${ }^{1}$ que se recogen en la Tabla 1.

Tabla 1

Criterios de inclusión de procedimientos quirúrgicos

No precisar preparación compleja

Escaso riesgo hemorrágico

Tiempo total de intervención inferior a 90 minutos

Dolor postoperatorio controlable con analgesia oral

No precisar drenajes con débito elevado

Tolerancia oral precoz

Que permita deambulación precoz 


\section{PACIENTES Y METODO}

Desde la incorporación de nuestro servicio a la Unidad de CMA (UCMA) del Hospital General Universitario de Valencia en enero de 2000 hasta abril de 2004, hemos realizado 21 corporoplastias con carácter ambulatorio, según la técnica de Nesbit, para la corrección de la incurvación peneana; en 12 pacientes con pene curvo congénito sin hipospadias y en 9 pacientes afectos de enfermedad de Peyronie. La edad media fue de 28,4 años (rango 15 - 63).

La indicación de la intervención la motivó la propia incurvación en 12 casos (57\%), la dificultad para la penetración en 7 (33\%) y en 2 casos (10\%) la imposibilidad de penetración.

Para objetivar el grado y tipo de curvatura, se empleó el método de autofotografía domiciliaria en erección propuesto por Kelami ${ }^{11}$.

En todos los pacientes se realizó la corrección quirúrgica de la incurvación mediante la siguiente secuencia operatoria:

1. Aplicación por el paciente en su domicilio, aproximadamente una hora antes de la intervención, de una crema de lidocaína y prilocaína al 50\% (crema EMLA), extendida tópicamente sobre toda la superficie del pene.

2. Infiltración troncular en la base del pene con anestésico local (lidocaína $2 \%$ )

3. Incisión coronal circunferencial asociada a postectomía en caso de prepucio redundante.

4. Exposición de la fascia de Buck tras disección roma de la piel hasta la base del pene.

5. Valoración del grado y dirección de la curvatura mediante erección artificial provocada por inyección intracavernosa de suero salino previa colocación de una banda compresiva en la raíz del pene (Fig. 1).

6. Incisión longitudinal a nivel lateral de la fascia de Buck llegando hasta la túnica albuginea.

7. Exéresis de uno o varios fragmentos elipsoidales de la túnica albugínea en la convexidad de la incurvación y posterior sutura contínua del defecto con ácido poliglicólico 3/0 (Dexon ${ }^{\circledR}$ ), procurando no lesionar el paquete vasculonervioso en las curvaturas ventrales ni la uretra en las dorsales.
8. Comprobación del grado de rectificación mediante nueva erección artificial, repitiendo el proceso en caso necesario hasta alcanzar una corrección aceptable (Fig. 2), pudiendo incluso participar el paciente en la aceptación del grado de enderezamiento.

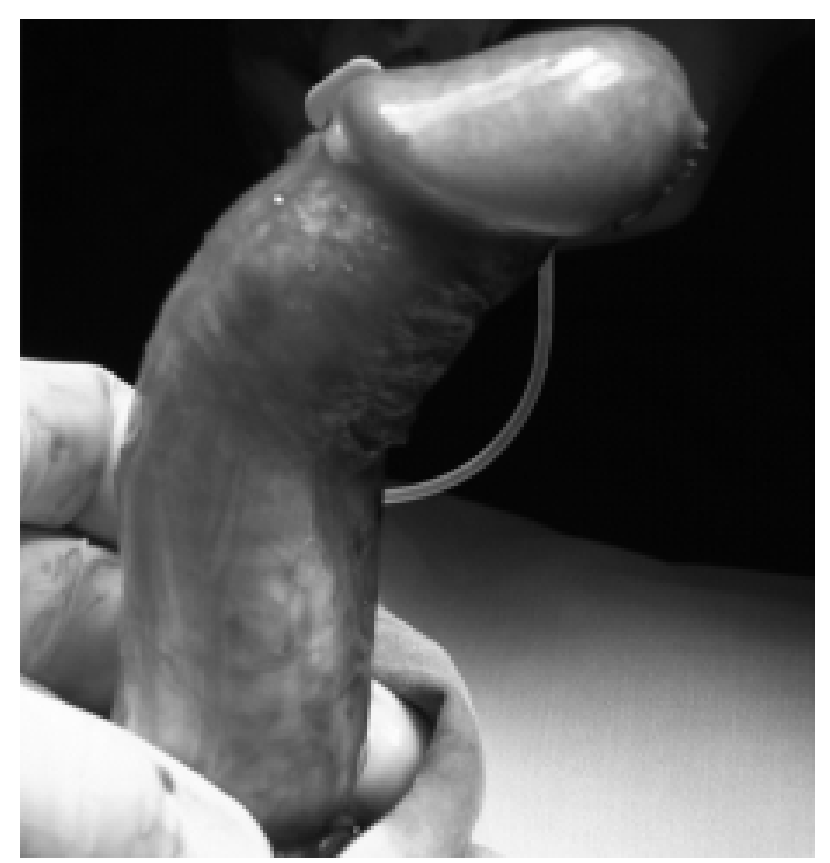

FIGURA 1. Valoración del grado y dirección de la curvatura mediante erección artificial.

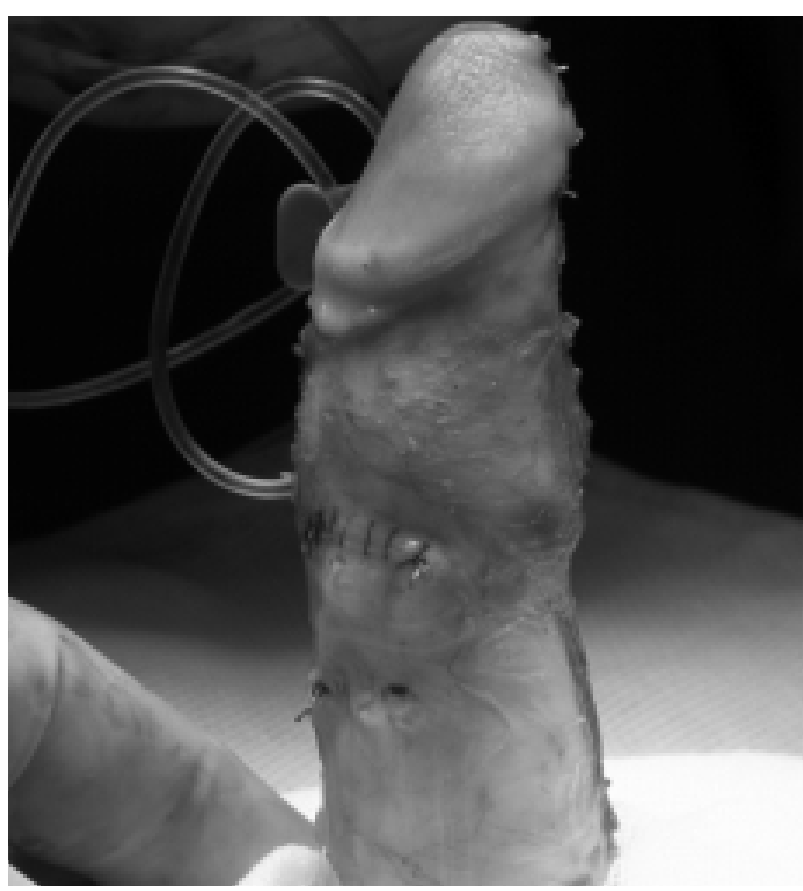

FIGURA 2. Comprobación del grado de rectificación tras la plastia. 
9. Cierre de la fascia de Buck con poligalactina 4/0 (Vicryl rapid®).

10. Sutura de la circuncisión con poligalactina $4 / 0$.

11. Sondaje uretrovesical con sonda de Foley $18 \mathrm{Ch}$. y vendaje compresivo, que se retiran a las 24 horas.

En todos los casos se realizó profilaxis antibiótica, utilizando una pauta monodosis (generalmente cefalosporina) previa al procedimiento quirúrgico.

El tratamiento postoperatorio consistió en la administración de analgésicos menores y diazepam, así como extremar las medidas higiénicas sobre la herida quirúrgica. También se les indicó abstinencia sexual durante 6 a 8 semanas posteriores a la intervención.

El seguimiento medio postquirúrgico fue de 27 meses (rango 3 - 51). En las revisiones hemos realizado una breve encuesta anónima sobre el grado de satisfacción del tratamiento recibido.

\section{RESULTADOS}

Todos los pacientes fueron intervenidos con anestesia local, no precisando en ningún caso sedoanalgesia adicional, no refiriendo dolor en ninguno de los distintos puntos de la secuencia operatoria descrita a excepción del paso de la sonda de Foley por la porción proximal de la uretra, que fue percibido por la práctica totalidad de los pacientes como un dolor tolerable de breve duración.

El tiempo medio utilizado en el procedimiento quirúrgico fue de 65 minutos (rango 50 - 75), siendo el número medio de incisiones en el cuerpo cavernoso de 24 (rango $1-5$ ).

Tras la intervención, los pacientes fueron trasladados a una sala de recuperación contigua al quirófano donde permanecieron un tiempo medio de 70 minutos (rango 60 - 90) acompañados de un familiar y controlados por personal de enfermería hasta que el paciente cumplió los criterios de alta que apuntamos en la Tabla 2 . Todos fueron dados de alta tras la intervención, no precisando ninguno de ellos ingreso hospitalario por complicaciones inmediatas intra ni postoperatorias.

En la revisión realizada una semana después de la intervención, todos los pacientes presenta-
Tabla 2

Criterios de alta

\begin{tabular}{|c|c|}
\hline - Consciente y orientado & - Control del dolor \\
\hline $\begin{array}{l}\text { - Constantes vitales estables } \\
\text { durante la última hora }\end{array}$ & $\begin{array}{l}\text { - Deambulación autónoma } \\
\text { sin sensación de } \\
\text { inestabilidad }\end{array}$ \\
\hline - Tolerancia de líquidos & - Ausencia de sangrado \\
\hline - Diuresis espontánea & $\begin{array}{l}\text { - Ausencia de nauseas y } \\
\text { vómitos }\end{array}$ \\
\hline
\end{tabular}

ban una evolución satisfactoria, contestando 20 de ellos (95\%) la encuesta sobre el tratamiento recibido en la UCMA, cuyos resultados se detallan en la Tabla 3.

En ningún paciente se constataron complicaciones locales inmediatas tales como edema prepucial, hematomas, infección de herida, necrosis de piel, etc. Las molestias en las zonas de sutura siempre fueron transitorias y en ningún caso constituyeron motivo de consulta por alteración estética.

En todos los pacientes se consiguió una buena corrección de la incurvación, no existiendo ninguna recidiva. Ningún paciente se mostró insatisfecho por el acortamiento peneano, lográndose en todos ellos unos buenos resultados, tanto estéticos como funcionales.

Tabla 3

Satisfacción con el tratamiento recibido

\begin{tabular}{lcc}
\hline Calificación & № casos & $\%$ \\
Excelente & 17 & $85 \%$ \\
Buena & 2 & $10 \%$ \\
Regular & 1 & $5 \%$ \\
Deficiente & 0 & $0 \%$ \\
\hline
\end{tabular}

\section{DISCUSION}

Las incurvaciones mínimas del pene en erección (menores de $15^{\circ}$ ) son muy frecuentes, careciendo de significado clínico siempre y cuando las erecciones sean indoloras y no dificulten las relaciones sexuales ${ }^{10}$. Cuando la curvatura es mayor de $30^{\circ}$ puede producir cierta dificultad para la relación sexual o molestias en la penetración, además de problemas psicológicos secundarios a la preocupación estética provocada por la incurvación de su pene en erección. 
Es fundamental evaluar el grado de curvatura y deformidad del pene, para lo que consideramos de gran importancia la realización de las autofotografías propugnadas por Kelami ${ }^{11}$, las cuales se efectúan en tres proyecciones durante una erección espontánea, permitiéndonos medir el ángulo de desviación y la calidad de la erección.

Todos nuestros pacientes aportaron autofotografias de suficiente calidad diagnóstica. No obstante, en caso de no poderlas obtener, algunos autores ${ }^{12}$ propugnan la inducción farmacológica de la erección con prostaglandina E1, siendo recomendable realizar también fotografias, ya que ello nos permite, además de realizar una completa evaluación, disponer de un documento gráfico de gran valor médico-legal.

El tratamiento quirúrgico de la incurvación del pene se basa fundamentalmente en tres tipos de técnicas:

1) Técnicas para alargar la túnica albugínea en la concavidad de la curvatura,

2) técnicas para acortar la túnica en la convexidad y

3) colocación de una prótesis.

Las primeras se fundamentan en la práctica de incisiones transversas en la concavidad de la incurvación o exéresis de la placa fibrosa en la enfermedad de Peyronie y colocación de parches de diversa procedencia (piel, safena, vena dorsal, dacron, goretex, duramadre, etc.); no obstante, se trata de una técnica actualmente muy poco utilizada por la frecuente aparición de disfunción eréctil postoperatoria y retracciones del injerto a medio-largo plazo $^{13,14}$. Asimismo, la colocación de prótesis se realiza como última opción terapéutica o bien se reserva para pacientes con disfunción eréctil inten$\mathrm{sa}^{14}$. Las técnicas orientadas a acortar la túnica albuginea en la convexidad de la curvatura, constituyen los procedimientos mas utilizados en la actualidad para el tratamiento de la incurvacion peneana, siendo la operación de Nesbit, y las múltiples variantes de plicatura descritas posteriormente, la técnica mas ampliamente difundida para el tratamiento de esta entidad, constituyendo actualmente la técnica original de Nesbit el "patrón oro" con el que se comparan todas las demás técnicas.

Existe controversia respecto al tipo de material de sutura (absorbible o no) a utilizar para reparar la túnica albuginea, ya que las suturas absorbibles pueden ser causa de reincurvación en caso de plicatura simple sin creación de zona cruenta. Por otra parte, además del inevitable acortamiento del pene, la complicación más frecuente de la plicatura sin excisión de la túnica albugínea es que puede producir abultamientos, a modo de "orejones", a nivel de la zona plicada. Asimismo, los puntos de sutura no absorbibles, a pesar de estar anudados de forma invaginante, pueden llegar a producir molestias durante la erección o el coito, e incluso provocar un problema estético al transparentarse a través de la piel. Así, en nuestra opinión, la escisión de la albugínea y sutura con material de reabsorción lenta proporciona unos excelentes resultados soslayando los inconvenientes que la protrusión de los nudos puede provocar y que a veces es necesario retirar cuando se utiliza material no absorbible ${ }^{12}$.

En cuanto a la discusión suscitada en relación a la utilización o no de sonda uretrovesical postoperatoria, aunque opinamos que es innecesario su empleo sistemático, consideramos que en CMA deben extremarse todas las medidas disponibles para minimizar riesgos de complicaciones. Así, un vendaje peneano muy apretado puede ser causa de retención urinaria, mientras que una venda demasiado holgada puede motivar la aparición de hematoma o edema. Por tanto, consideramos que la colocación de sonda y vendaje compresivo durante las primeras 24 horas del postoperatorio soslaya dichas complicaciones conllevando una mínima morbilidad.

Por otro lado, es un hecho que en los últimos años la CMA avanza de forma imparable; así en Estados Unidos, país donde más se ha desarrollado este régimen asistencial, la CMA supone cerca del $60 \%$ de todas las intervenciones realizadas $^{4,15}$. Asimismo, son cada vez más frecuentes los trabajos divulgados en las revistas de urología de nuestro país acerca de este sistema organizativo de la asistencia quirúrgica ${ }^{16}$, lo que pone de manifiesto que la urología es una especialidad con gran proyección en el campo de la CMA.

Coincidimos con la idea de Llopis y $\operatorname{cols}^{17}$ en que la CMA constituye un sistema asistencial en el que todos los participantes se benefician. En efecto, comporta un beneficio para el hospital ya que su utilización implica una reducción del costo sanitario, que varía entre el 25 y $75 \%$ según diversos autores ${ }^{18-20}$, pues con la ambulatorización del proceso, aunque el coste de la intervención sea 
semejante al proceso con ingreso, el ahorro se produce fundamentalmente en hostelería y en personal sanitario. Asimismo, el hospital también se beneficia al aumentar el número de camas disponibles, ya que según nuestra propia experiencia y la de otros autores revisados, la estancia hospitalaria media de los pacientes intervenidos mediante técnica de Nesbit con ingreso varía entre 3 y 3,5 días $^{10,21}$.

En lo que concierne al paciente, su modo de vida se altera mínimamente, ya que vuelve a su domicilio a las pocas horas de ser intervenido, reduciéndose ostensiblemente su grado de ansiedad así como la probabilidad de complicaciones propias de la hospitalización, tales como infecciones nosocomiales y complicaciones secundarias al encamamiento ${ }^{22}$. Además, su grado de satisfacción suele ser elevado, tal y como demuestra nuestra encuesta, y que se sitúa entorno al $95 \%$.

De igual forma, creemos que el cirujano también se beneficia, pues aunque al principio la CMA pueda ser considerada como una cirugía de segunda línea, el urólogo, pronto se ve implicado en un sistema de trabajo donde "todo vale" para minimizar riesgos, desde la aplicación de crema de lidocaína y prilocaína 1 hora antes de la intervención para anestesiar la piel, la metódica infiltración con anestésico local, la utilización de incisiones justas, hemostasia rigurosa, así como obviar gestos quirúrgicos innecesarios para abreviar el tiempo operatorio, etc., suponen un reto profesional, que al menos para nosotros ha resultado enriquecedor y gratificante. Igualmente, supone una mayor motivación profesional a la vez que un mayor índice de satisfacción a la vista de los resultados obtenidos. Hemos tenido una nula incidencia de complicaciones a excepción del inevitable acortamiento del pene (habitualmente carente de significado clínico y legal si se efectúa previamente una información exhaustiva y se dispone de documento de consentimiento), claramente independiente del régimen de ambulatorización o no del proceso. Todo ello implica que actualmente en nuestro hospital, la práctica totalidad de correcciones de incurvación peneana son realizadas sin necesidad de ingreso. Igualmente, y según vamos adquiriendo más experiencia, vamos ampliando nuestra cartera de procedimientos, incluyendo patologías de mayor complejidad siendo cada vez menos selectivos en lo que al riesgo quirúrgico se refiere.

\section{REFERENCIAS}

1. Ministerio de Sanidad y Consumo. Dirección General de Aseguramiento y Planificación Sanitaria. Cirugia Mayor Ambulatoria: Guía de Organización y Funcionamiento. Madrid, 1993.

2. De Lathower C, Pouillier JP. How much ambulatory surgery in the world in 1966 - 1977 and trends. Amb. Surg 2000;8:191-195.

3. Jarret P, De Lathower C, Ogg TW. The time has come to promote true day surgery. Amb. Surg 2000;8:163-167.

4. Rodríguez JM, Rodríguez R, Blanco G. Cirugía Mayor Ambulatoria en Urología. En: Porrero JL. Cirugía Mayor Ambulatoria 2a Edición; Ed Doyma SA; Madrid 2002;311-333.

5. Navalón P, Zaragoza C, Canovas JA, et al. Cirugía Mayor Ambulatoria en Urología. Análisis de nuestra experiencia. Arch Esp Urol 2004;57:513-518.

6. Generalitat Valenciana. Conselleria de Sanitat. Guía de actuación en Cirugia Mayor Ambulatoria. Valencia, 2002.

7. Nesbit RM. Congenital curvature of the phallus: report of 3 cases with description of corrective operation. J Urol 1965;93:230-325.

8. Essed E, Schoroeder FH. New surgical treatment for Peyronie's disease. Urology 1985;25:282-288.

9. Erpenbach K, Rothe H, Derschum W. The penile plication procedure. An alternative method for straightening penile deviation. $\mathrm{J}$ Urol 1991;146:1276-1278.

10. Benejam J, Rodriguez Vela L, Garcia Miralles R, et al. Tratamiento con plicatura modificada de la túnica albuginea en pacientes con incurvación peneana congénita. Actas Urol Esp 1999;23:36-42.

11. Kelami A. Autophotography in evaluation of functional penile disorders. Urology 1983;21:628-629.

12. Rodríguez Vela L, Chantada V, Fiter L, et al: Incurvación congénita del pene. En: Martín Morales A, Chantada V, Fiter L y cols. Enfermedad de La Peyronie y otras alteraciones morfométricas del pene; Ed. Ene SA; Madrid 2001;101-119.

13. Moncada I. Incurvaciones peneanas congénitas y adquiridas. Enfermedad de Peyronie. En: Saénz de Tejada I, Allona A. Erección, eyaculación y sus trastornos; Ed. Fomento Salud SL; Madrid 1997;223-256.

14. Gholami SS, Lue TF. Enfermedad de Peyronie. En: Lue TF. Clínicas Urológicas de Norteamérica; McGraw Hill; Madrid 2001;401-416.

15. Davis JE. Futuro de la Cirugía mayor ambulatoria. Clin Quir Nort 1987;4:921-927.

16. Elizande A, Regojo O, Navarro J, et al. Urologia en un programa de Cirugia Mayor Ambulatoria. Actas Urol Esp 2004;28(5 supl):144.

17. Llopis B, Navarro JA, Mola MJ, et al. Cirugía mayor ambulatoria en urología: 5 años de experiencia. Actas Urol Esp 2003;27:117-122.

18. Caldamone A, Rabinowitz R. Outpatient orchiopexy. J Urol 1990;143:951-956.

19. Sadler G, Richards H, Watkins G, et al: Day-case paediatric surgery: the only choice. Ann R Coll Surg Engl 1982;74:130-135.

20. Vargas C, Rius G. Cirugía urológica sin ingreso. Actas Urol Esp 1993; 17:87-91.

21. Rodríguez J, Franco E, Prats JM, et al. Tratamiento quirúrgico de la incurvación congénita del pene mediante técnica de Nesbit. Arch Esp Urol 2004;57:141-145.

22. Sampietro A, Vaquerizo A, Fernández A, et al. Cirugía mayor ambulatoria en Urologia: nuestra experiencia. Arch Esp Urol 1995;48:343-347.

Dr. P. Navalón Verdejo

Paseo Alameda 38-2

46023. Valencia.

E-mail: pnavalonverdejo@hotmail.com

(Trabajo recibido el 14 julio de 2004) 